\title{
Was sind Risikofaktoren für CED?
}

- Chronisch-entzündliche Darmerkrankungen (CED) nehmen stetig zu. Vor allem in den letzten zehn Jahren konnte bei Kindern ein deutlichen Anstieg v.a. von Morbus Crohn (MC) verzeichnet werden. „Es müssen Umweltfaktoren sein, die gerade in den Industrienationen und inzwischen auch in den Schwellenländern zu einem Anstieg dieser Erkrankungen führen“, sagte Prof. Dr. Sibylle Koletzko aus München. Vor allem Rauchen erhöht das Risiko, besonders für einen $M C$ - sowohl für das Auftreten als auch für die Prognose des Patienten. „Mit der wichtigste Faktor für eine schlechte Prognose ist das Rauchen. Das ist viel wichtiger als die Medikamente, die eingenommen werden“, erklärte Koletzko. Sie fand außerdem in einer Studie heraus, dass Kinder, die nie gestillt wurden, ein um das 3-4-fach erhöhtes Risiko für einen MC hatten.
Auch die Hygiene-Hypothese könnte für CED gültig sein. Sie besagt, dass die Abnahme von Infektionen gleichzeitig mit einem Anstieg von verschiedenen immunologisch bedingten Erkrankungen, wie Allergien, Multiple Sklerose und Typ-1-Diabetes, assoziiert ist. „Und dazu gehört auch der Morbus Crohn“, stellte Koletzko fest. Und schließlich konnte ein „Bauernhofeffekt“ belegt werden: Sowohl MC als auch Colitis ulcerosa (CU) findet man häufiger in der Stadt als auf dem Land. Bei Kindern, die Kontakt zu Stalltieren haben, senkt sich das Risiko für MC um die Hälfte, für CU um 60\%. Patienten mit MC leiden außerdem häufiger unter einer allergischer Rhinitis. Zum Schluss wurden auch noch protektive Faktoren gefunden: Für eine $\mathrm{CU}$ ist dies eine durchgemachte Appendizitis oder Lymphadenitis, nicht jedoch eine Appendektomie. „Es kommt jedoch nur zu

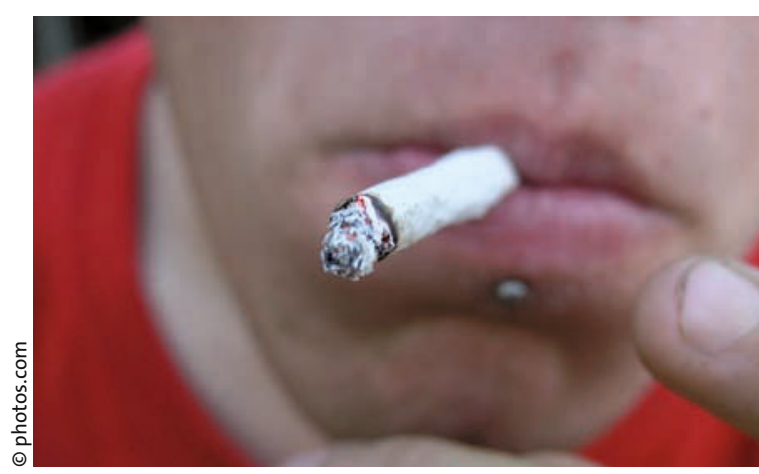

einer Risikoreduktion für die CU, wenn der Blinddarm in der Kindheit entfernt wurde", fügte Koletzko hinzu. Außerdem konnte belegt werden, dass der Verzehr von Ballaststoffen, Fisch und Omega-3-Fettsäuren protektiv wirkt.

nz

Koletzko S. Epidemiologie von chronisch entzündlichen Darmerkrankungen (CED) in Industrienationen

\section{Mit Diabetes länger warten auf die Pubertät}

— Zu Spätentwicklern in Sachen Pubertät scheint ein Diabetes mellitus Typ 1 betroffene Kinder zu machen. Wie ausgeprägt dieser Effekt ist, zeigt eine Auswertung der Diabetes-Patienten-Verlaufsdaten (DPV), die Dr. Tilman Rohrer, Homburg/Saar, vorstellte: Bei erkrankten Jungen beginnt die Genitalentwicklung im Mittel erst mit 12,0 Jahren, die ersten Schamhaare sprießen im Alter von 12,2 (normal: 11,1 bzw. 11,8). Junge Diabetikerinnen sind mit 11,4 Jahren signifikant älter als nicht erkrankte Mädchen
$(10,9)$, wenn die Brustentwicklung startet. Das Menarchealter liegt bei 13,2 Jahren versus 12,7 in der Normalbevölkerung. Diesen Anlaufschwierigkeiten folgt allerdings ein erfolgreicher Spurt: Das Tanner-Stadium 5 erreichen die zuckerkranken Jugendlichen in normalem Alter. Wie stark der einzelne junge Diabetiker mit der Pubertätsentwicklung hinterherhinkt, hängt dabei von verschiedenen Faktoren ab, erklärte Rohrer. Entscheidend ist die Einstellung des Blutzuckers: Je höher der $\mathrm{HbA}_{1 c}$-Wert, desto später kommen die Kinder in die Pubertät und desto höher ist das Menarchealter. Zudem spielt auch bei zuckerkranken Kindern das Körpergewicht eine Rolle - mit Abnahme des BMI verzögert sich die Pubertätsentwicklung weiter. Eine Verzögerung der Menarche wird darüber hinaus umso wahrscheinlicher, je länger die relative Diabetesdauer zu Beginn der Pubertätsentwicklung ist.

bs

Rohrer T. Diabetes in Pubertät und Entwicklung

\section{PTT: Auf altersadaptierte Normwerte achten}

— Eine verlängerte partielle Thromboplastinzeit (PTT) macht noch keine Hämophilie aus und eine normale PTT schließt kein VonWillebrand-Syndrom aus. Diese zwei Statements hat PD Dr. Hans-Jürgen Laws aus Düsseldorf seinen Zuhörern als „, take home message" mitgegeben. Er betonte, dass bei der Bestimmung des PTT unbedingt altersadaptierte Normwerte verwendet werden sollten. „Ein Teil unserer Zuweisungen ergibt sich daraus, dass Erwachsenennormwerte herangezogen werden." Die Staffe- lung ist gerade in den ersten drei Lebensmonaten ganz erheblich. Laws gab Werte von 42,9 (31-54) s für den 1.-3. Lebenstag und 37,1 (29 bis 50) s für den 2.-4. Lebensmonat an. Und zu einem jungen Erwachsenen sind die Unterschiede noch einmal größer: Für Kinder zwischen 1 und 14 Jahre liegen sie bei 31 (28-39) s. Die Normwerte sind außerdem von Labor zu Labor verschieden.

Neben falschen Normwerten kommen noch andere Fehlerquellen infrage, die zu Schwan- kungen bei der Bestimmung der PTT führen können. Laws fasste die Top Ten der präanalytischen Fehlerquellen zusammen. Neben den schon erwähnten falschen Normwerten sind dies: lange Stauung, kleine Kanüle, schlechte Abnahme (z. B. Hämatome), ,'Stochern" (Rollvenen), Stress des Kindes, Röhrchen unzureichend gefüllt, schaumige Abnahme, Lagerung (auch im Kühlschrank) und zu lange Transport- bzw. Lagerungszeiten, die max. vier Stunden betragen sollen. $\mathbf{n z}$

Laws H.-J. Präoperative Gerinnungsdiagnostik - was ist zu empfehlen? 